In Cres. Vol. $4 N^{\circ} 1:$ pp. 103-112, 2013

\title{
EFECTO SOBRE EL MÚSCULO LISO INTESTINAL Y TOXICIDAD AGUDA ORAL DE UN EXTRACTO DE CHILCA (Baccharis latifolia) ${ }^{*}$
}

\author{
EFFECT ON INTESTINAL SMOOTH MUSCLE AND \\ ORAL ACUTE TOXICITY OF AN EXTRACT \\ OF CHILCA (Baccharis latifolia)
}

\author{
Liz Elva Zevallos Escobar, \\ Jorge Luis Arroyo Acebedo ${ }^{2}$
}

\section{RESUMEN}

El objetivo fue evaluar el efecto de un extracto hidroalcohólico del Baccharis latifolia sobre el músculo liso intestinal y toxicidad aguda oral. Material y métodos: se evaluó el efecto del extracto en el modelo in Vitro de íleon aislado de cuy estimulado con acetilcolina e histamina $10^{-3} \mathrm{M}$, usando atropina y hioscina como fármacos de referencia. La toxicidad aguda oral se determinó mediante la dosis letal media $\left(\mathrm{DL}_{50}\right)$ en ratones. Resultados: el extracto presenta relajación del músculo liso intestinal a una $\mathrm{CE}_{50}$ de $46,41 \mathrm{mg} / \mathrm{mL}$ y una estimulación del músculo a una CE de $182,2 \mathrm{mg} / \mathrm{mL}$. La $\mathrm{DL}_{50}$ fue de $2000 \mathrm{mg} / \mathrm{kg}$. Conclusión: El extracto presenta una actividad bifásica de tipo hormesis, que se manifiesta por una relajación prolongada del músculo liso a bajas concentraciones, seguida de un efecto espasmogénico a altas concentraciones. No se encontraron alteraciones toxicológicas.

PALABRAS ClAVE: Actividad bifásica, Baccharis latifolia, hormesis, íleon aislado, músculo liso intestinal, toxicidad aguda oral.

* Recibido: 19 de marzo del 2013; aprobado: 31 de marzo del 2013.

1 Maestra en Farmacología. Docente de la Escuela profesional de Farmacia y Bioquímica ULADECH Católica. E-mail: lzevallose@uladech.pe

2 Doctor en Farmacia. Docente de la Facultad de Medicina Humana UNMSM. Docente de la Unidad de Post-Grado de Farmacia y Bioquímica UNMSM. 


\begin{abstract}
The objective was to evaluate the effect of a hydroalcoholic extract of Baccharis latifolia on intestinal smooth muscle and acute oral toxicity. Material and Methods: The effect of the extract on the in vitro model of isolated guinea pig ileum stimulated with acetylcholine and histamine $10^{-3} \mathrm{M}$ was evaluated, using atropine and hyoscine as reference drugs. The acute oral toxicity was determined by the median lethal dose $\left(\mathrm{LD}_{50}\right)$ in mice. Results: the extract has relaxation of intestinal smooth muscle to an $\mathrm{EC}_{50}$ of $46,41 \mathrm{mg} / \mathrm{mL}$ and muscle stimulation at an $\mathrm{EC}$ of $182,2 \mathrm{mg} / \mathrm{mL}$. The $\mathrm{LD}_{50}$ was $2000 \mathrm{mg} / \mathrm{kg}$. Conclusion: The extract has an activity hormesis biphasic type, manifested by a prolonged relaxation of smooth muscle at low concentrations followed by a spasmogenic effect at high concentrations. There were no toxicological alterations.
\end{abstract}

KEY WORDS: Biphasic activity, Baccharis latifolia, hormesis, isolated ileum, intestinal smooth muscle, acute oral toxicity.

\title{
I. INTRODUCCIÓN
}

El reino vegetal representa un enorme potencial de moléculas para ser descubiertas, ya que se estima que más del $90 \%$ de las especies vegetales no han sido exhaustivamente estudiadas. El Perú es privilegiado con las condiciones climáticas, cuenta con plantas con propiedades medicinales, las cuales no se han difundido debido al escaso conocimiento científico que se tiene de ellas, son usadas como remedios no oficiales y circulan solo por la reputación que el uso popular les ha conferido. ${ }^{1-3}$

La especie Baccharis latifolia se utiliza por la población en forma de infusión por su efecto relajante en el músculo liso intestinal, hecho que motivó el estudio del efecto del extracto hidroalcohólico de la planta sobre las contracciones inducidas por dos espasmógenos en órganos aislados. ${ }^{4,5}$

Es necesario profundizar en el estudio científico del potencial terapéutico de las especies vegetales utilizadas en la medicina popular y más importante aún valorar su efectividad e inocuidad. ${ }^{6}$

\section{PROBLEMA}

¿Cuál es el efecto sobre el músculo liso intestinal y cuál el efecto tóxico de un extracto de Chilca (Baccharis latifolia)?

\section{HIPÓTESIS}

El extracto de Chilca tiene efecto relajante sobre el músculo liso intestinal y no es tóxico en su administración. 


\section{OBJETIVO}

Evaluar el efecto sobre el músculo liso intestinal del íleon de cobayo de un extracto de Chilca (Baccharis latifolia) y valorar la toxicidad aguda oral.

\section{MATERIALES Y MÉTODO}

\section{Preparación del extracto según Lock}

La planta fue recolectada en el distrito de La Unión, provincia de Dos de Mayo, Departamento de Huánuco, e identificadas en el museo de historia natural de la Universidad Nacional Mayor de San Marcos (UNMSM); se pulverizaron y secaron a $40{ }^{\circ} \mathrm{C}$ y se sometió a extracción con 1 litro de etanol al $80 \%$. El extracto se filtró y concentró en un evaporador rotatorio.

\section{Estudio fitoquímico preliminar del extracto hidroalcohólico según Lock ${ }^{7}$}

La detección de metabolitos secundarios se realizó mediante pruebas químicas de caracterización (reacción de shinoda, tricloruro férrico, con la gelatina, prueba de la espuma, dragendorff, mayer, molish, lieberman- burchard).

\section{MODELO EXPERIMENTAL}

\section{Íleon aislado de cobayo in vitro ${ }^{8}$}

Se utilizaron cobayos procedentes del INS, privados de alimentos las primeras $24 \mathrm{~h}$, se sacrificaron para una laparotomía y se extrajo un segmento de íleon sumergido en solución Tyrode a $37{ }^{\circ} \mathrm{C}$ en baño para órgano aislado, burbujeada con una mezcla de $95 \%$ de $\mathrm{O}_{2} \mathrm{y} 5 \%$ de $\mathrm{CO}_{2}$. Los segmentos se acoplaron a un transductor de fuerza de desplazamiento conectado a un quimógrafo y se registraron las contracciones, para determinar la amplitud de cada contracción, midiendo el desplazamiento, en centímetros, producido por la respuesta contráctil inducida por el espasmógeno a partir de la línea de base.

Las variables fueron amplitud de la contracción y frecuencia.

\section{Actividad sobre el músculo liso intestinal}

Se observó el cambio de tono del músculo liso del íleon precontraído con los espasmógenos acetilcolina e histamina $10^{-3} \mathrm{M}$, se ensayaron diferentes concentraciones del extracto $90,125,150,200$ y $250 \mathrm{mg} / \mathrm{mL}$, se comparó con atropina $10^{-3} \mathrm{M}$ y Hioscina $10^{-3} \mathrm{M}$ como referencia. Se consideró como el $100 \%$ de respuesta a la mayor amplitud en el tono muscular alcanzada al emplear los 
espasmógenos y se calcularon los valores relativos, en porcientos, de las restantes respuestas dadas por el extracto a diferentes concentraciones.

\section{Toxicidad aguda oral según la $\mathrm{OMS}^{9}$}

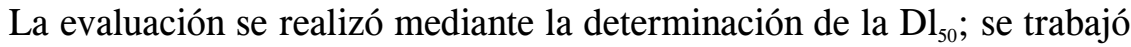
con 32 ratones de raza albina entre 20 y $34 \mathrm{~g}$ de ambos sexos, mantenidos a temperatura controlada; se les estandarizó su alimentación y agua ad libitum. La administración del extracto se realizó por vía oral previo ayuno de 14 horas. Aleatoriamente se formaron grupos experimentales y de control, constituido cada uno por 08 animales, a los que se les administró el extracto a dosis de 500, 1000,1500 y $2000 \mathrm{mg} / \mathrm{kg}$ de peso, además de un grupo de control con solución salina, siendo observados constantemente durante las primeras 24 horas y diariamente en un período de 14 días, registrando cualquier síntoma tóxico. Al finalizar el período, se procedió al sacrificio para realizar la necropsia y un examen macroscópico y posteriormente microscópico de órganos y tejidos. El valor de la dosis letal 50 se estimó mediante el método estadístico de Probits.

\section{ANÁLISIS ESTADÍSTICO}

Los resultados fueron presentados en tablas y gráficos y sometidos a un ANVA y un análisis con " $\mathrm{t}$ " de student, para comprobar las diferencias entre las medias. Se tomó como nivel de significación $\mathrm{p}<0,05$.

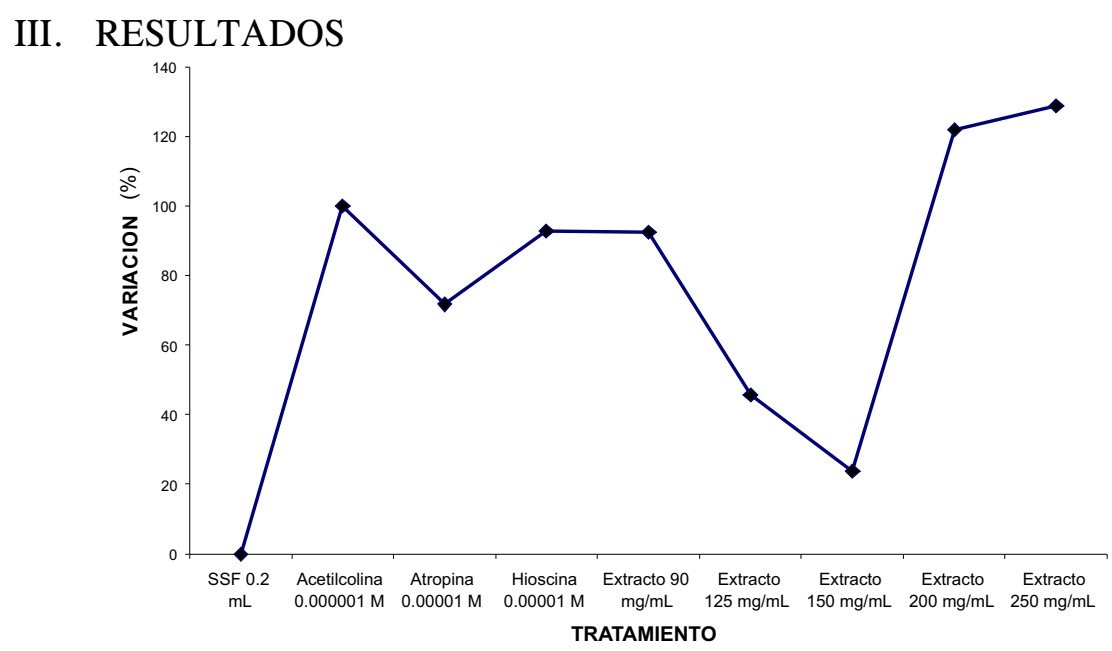

Figura 01. Porcentaje de variación del efecto sobre el músculo liso intestinal del extracto hidroalcohólico de Chilca (B. latifolia) sobre la contracción del íleon de cobayo inducida por acetilcolina. 
Efecto sobre el músculo liso intestinal y toxicidad aguda oral de un extracto de Chilca

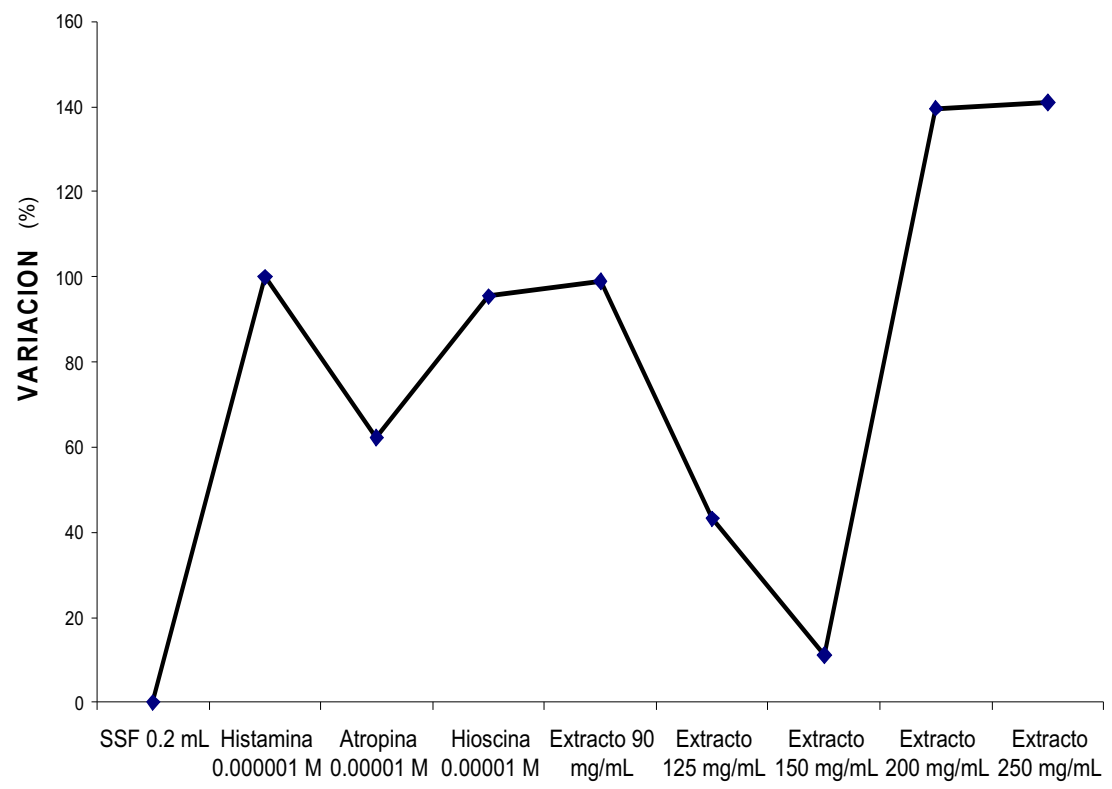

TRATAMIENTO

Figura 02. Porcentaje de variación del efecto sobre el músculo liso intestinal del extracto de Chilca (Baccharis latifolia) sobre la contracción del íleon de cobayo inducida por histamina.
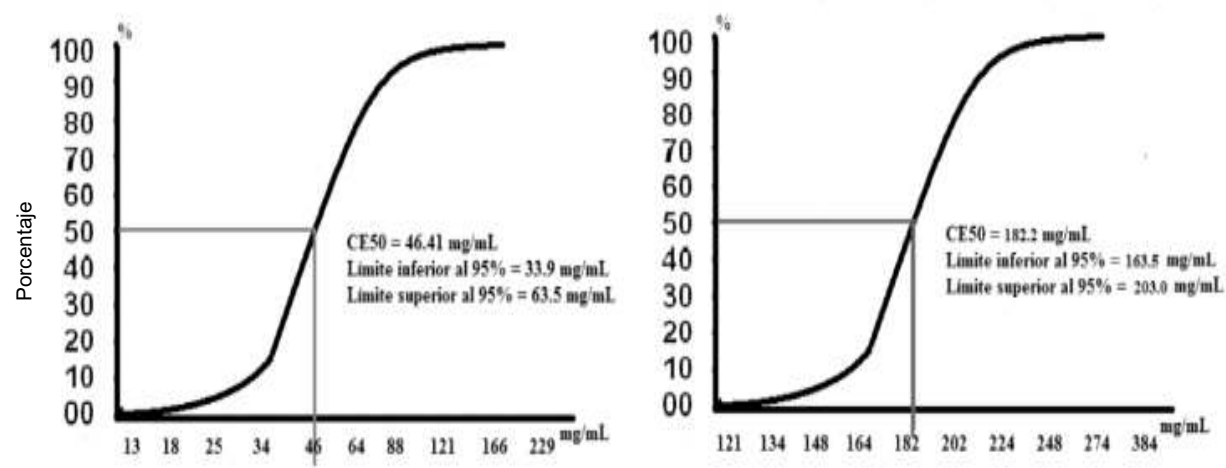

Figura 03. Concentración efectiva media $\left(\mathrm{CE}_{50}\right)$ del extracto de Chilca (Baccharis latifolia) para inducir relajación y estimulación del íleon de cobayo. 
Tabla 01

SIGNOS CLÍNICOS DE LA EVALUACIÓN TOXICOLÓGICA EN LOS ANIMALES PROBLEMA DESPUÉS DE LA ADMINISTRACIÓN DEL EXTRACTO DE CHILCA (Baccharis latifolia)

\begin{tabular}{|c|c|}
\hline Periodo de observación & Signos clínicos \\
\hline Media hora después de la administración & Ligero letargo y somnolencia \\
\hline A partir de las 3 horas de la administración & Signos de recuperación \\
\hline A partir de cuatro horas de la administración & $\begin{array}{l}\text { Reflejo postural normal, hábitos de } \\
\text { aseo y consumo de alimento y agua } \\
\text { normales }\end{array}$ \\
\hline Segundo día & $\begin{array}{l}\text { Agrupación intermitente en los } \\
\text { extremos de la jaula }\end{array}$ \\
\hline Desde el tercer día, hasta el final del estudio & $\begin{array}{l}\text { No se apreciaron signos de toxicidad, } \\
\text { se mostraron normales en su } \\
\text { comportamiento }\end{array}$ \\
\hline
\end{tabular}

\section{DISCUSIÓN}

La marcha fitoquímica preliminar muestra gran cantidad de flavonides, compuestos fenólicos, alcaloides y compuestos terpénicos. La información recogida según estudios etnofarmacológicos, farmacológicos, toxicológicos y fitoquímicos, realizados en el género Baccharis describe algunos de sus componentes químicos, tales como alcaloides, flavonoides y otros compuestos fenóli$\cos$, triterpenoides y aceites volátiles. Algunos estudios proponen que los flavonoides y los alcaloides como fitoconstituyentes de plantas medicinales, han demostrado tener efecto relajante en el músculo liso en diferentes modelos experimentales. ${ }^{11-14}$

El extracto de Chilca (Baccharis latifolia), a diferentes concentraciones, fue capaz de relajar el músculo liso del intestino frente al efecto contráctil de acetilcolina (Ach), el efecto relajante fue significativamente mayor a concentraciones de 125 y $150 \mathrm{mg} / \mathrm{mL}$ donde se observa una actividad de relajación que corresponde a 45,74 y 23,73 \% de variación, respectivamente (Ver figura 01).

De manera similar se realizó el mismo experimento con histamina donde se pudo observar que el extracto a concentraciones de $125 \mathrm{y} 150 \mathrm{mg} / \mathrm{mL}$ produjo una mayor actividad de relajación con un 43,17 y 11,15 \% de variación frente al efecto contráctil de histamina sobre el tejido intestinal (Ver figura 02). 
La disminución del tono muscular intestinal con el extracto posterior a la contracción inducida con Ach e histamina se debe, de alguna manera, a la alteración o inhibición de alguno de los pasos de la contracción inducida por canales que dependen del voltaje. Esto sugiere que el $B$. latilfolia posee un mecanismo de acción alterno al de atropina o hioscina. Aunque se conocen diversos mecanismos farmacológicos que explican el mecanismo de acción de los compuestos que relajan la musculatura lisa intestinal conocidos como antiespasmódicos, el más común es la actividad como antagonista competitivo de la Ach y, con ello, impiden la despolarización de la célula muscular y su consiguiente contracción. ${ }^{15,16}$

La atropina es el fármaco más potente del grupo de los compuestos antiespasmódicos, indicados para el tratamiento de cólico intestinal por relajar el músculo liso intestinal. El ensayo para el efecto contráctil de Ach en presencia de atropina mostró una respuesta de relajación moderada con un 71,75\% de variación y para el efecto contráctil con histamina mostró una relajación de $62,23 \%$ de variación. El estudio mostró que la hioscina inhibió el efecto contráctil de Ach en 92,83\% de variación y el efecto contráctil de histamina en 95,32 \% de variación, respecto a la contracción inducida, resultados que muestran un disminuido efecto relajante sobre el músculo liso intestinal en relación con la actividad relajante de los extractos (Figuras 01 y 02). Diferentes estudios han mostrado una acción relajante de atropina y hioscina cuando el íleon aislado se contrae con Ach e histamina. ${ }^{15-18}$

El extracto del Baccharis latifolia a concentraciones de 200 y $250 \mathrm{mg} / \mathrm{mL}$ provocaron un efecto de rebote que generó contracción en el músculo liso intestinal superior al provocado por los espasmógenos llegando a 121,97 y 128,7 \% de variación para los extractos, respectivamente, cuando se trabajó con Ach y cuando se trabajó con histamina 139,57 y 141,01\% de variación. El efecto estimulante del extracto a concentraciones altas significaría una respuesta de compensación que actuarían como agonistas de los receptores M3 y H1, prolongando la actividad contráctil de Ach e histamina, es decir un sinergismo. ${ }^{16,19}$

Los resultados muestran que el extracto del B. latifolia, dependiente de las concentraciones, presenta una actividad bifásica en el íleon de cobayo aislado, que se manifiesta por una relajación prolongada que inhibe la respuesta contráctil inducida por Ach e histamina, resultados que están básicamente de acuerdo con otros descritos previamente para el género baccharis y una estimulación de la contracción del músculo liso intestinal dependiente de su concentración que no se ha reportado en algún otro estudio del género baccharis. 
El efecto relajante del extracto dependió claramente de las concentraciones utilizadas, considerándose una $\mathrm{CE}_{50}$ de $46,41 \mathrm{mg} / \mathrm{mL}$ del extracto del Baccharis latifolia, para inducir relajación del músculo liso; y, además, se consideró una $\mathrm{CE}_{50}$ de $182,2 \mathrm{mg} / \mathrm{mL}$ del extracto hidroalcohólico para inducir estimulación del músculo liso (Figura 03).

La presente investigación muestra efectos contrarios al emplear el extracto en concentraciones bajas y altas; esto posiblemente tendría una explicación científica, tipo hormesis. Los reportes científicos definen a la hormesis cuando las sustancias en dosis pequeñas tienen efectos diferentes u opuestos a cuando se utilizan dosis mayores. ${ }^{20,21}$

Al evaluar el efecto toxicológico del Baccharis latifolia, se observó que la administración del extracto a diferentes dosis y a una dosis límite de 2000 $\mathrm{mg} / \mathrm{kg}$ de masa corporal no provocó muerte en los animales de los grupos problema (Tabla 01). Los signos aparecidos en el grupo problema después de la administración de la sustancia de ensayo (letargo y somnolencia) sugieren toxicidad; sin embargo, la pronta recuperación sugiere una intoxicación ligera o leve. En el grupo control no se evidenciaron los signos clínicos observados en los animales tratados; sólo se observó un estado de estrés atribuido a la manipulación. En nuestro estudio de toxicidad oral aguda, no se observó cambios significativos en la ganancia o pérdida de peso, ni alteraciones patológicas en órganos y tejidos, que puedan ser atribuidas a la administración del extracto. Debido a la ausencia de mortalidad o presencia de animales moribundos, se puede afirmar que la $\mathrm{DL}_{50}$ oral para el extracto es superior a $2000 \mathrm{mg} / \mathrm{kg}$, calificándose, según el Sistema Global Armonizado, como "no clasificada" o "no tóxica". ${ }^{11}$

Cabe señalar que en un estudio de la Universidad Nacional de Colombia, donde se realizaron pruebas de toxicidad aguda oral en ratas de la especie $B$. latifolia, por administración del extracto etanólico a dosis de $2000 \mathrm{mg} / \mathrm{kg}$, los hallazgos clínicos más representativos fueron ligera depresión y presencia de heces blandas. También se realizó el estudio de toxicidad subaguda oral en ratas, de lo cual, se concluyó que la administración diaria por 28 días del extracto etanólico, dosificado a $1000 \mathrm{mg} / \mathrm{kg}$, no ocasiona mortalidad, pero sí alteraciones en el peso relativo de órganos como el bazo y los riñones, así como lesiones en tejido renal, que pueden sugerir una leve respuesta tóxica. ${ }^{22}$

En este sentido, el efecto relajante sobre el músculo liso intestinal, atribuida a la especie Baccharis latifolia, valida su acción relajante sobre el músculo liso intestinal, referida en la medicina popular y se corresponde con lo hallado 
experimentalmente, teniendo en cuenta las bajas concentraciones, ya que en dosis altas causa un efecto de rebote produciendo una actividad contráctil. En cuanto al efecto tóxico, por no ser letal se considera no tóxico.

\section{CONCLUSIÓN}

El extracto de Chilca (Baccharis latifolia) sobre el músculo liso intestinal ha demostrado un efecto bifásico, conocido como de tipo hormesis, donde en concentraciones bajas $\left(\mathrm{CE}_{50}\right)$ de $46,41 \mathrm{mg} / \mathrm{mL}$, brinda un efecto relajante; y en dosis altas (CE§) 182,2 mg/mL, un incremento de la contracción del íleon de cobayo. En las condiciones experimentales, la administración oral del extracto en ratones no mostró toxicidad, la $\mathrm{DL}_{50}$ se encuentra por encima de $2000 \mathrm{mg} / \mathrm{kg}$ de masa corporal, calificándose así como "no tóxica".

\section{REFERENCIAS BIBLIOGRÁFICAS}

1 HERNÁNDEZ A. 2005. Fitoterapia. Bases científicas y legales para su aplicación. BLACPMA. 4(4): 71-85.

2 KUKLINSKI C. 2003. Farmacognosia. Ediciones Omega.

3 Freire S, Urtubey E, Giuliano D. 2007. Epidermical characters of baccharis (asteraceae) species used in traditional medicine. Caldasia. 29(1):23-38.

4 Beltrán H, Granda A, León B, Sagástegui A, Sánchez I, Zapata M. 2006. Astereaceas endémicas del Perú. Rev. peru. Biol. 13(2): 64 -164.

5 HoYOS K, YIEN YEP M. 2008. Diseño de una formulación de aplicación tópica a base de Baccharis latifolia (Chilca), con efecto antiinflamatorio [Tesis]. Universidad Nacional Mayor de San Marcos. Perú.

6 SERRANO L. 2005. Actividad antiespamódica de extractos de plantas medicinales en preparaciones de íleon de cobayo. [Tesis]. Universidad Autónoma de Nuevo León. México.

7 LOCK DE UGAZ, O. 1994. Investigación fitoquímica: Métodos en el estudio de productos naturales. Segunda edición. Fondo editorial Pontificia Universidad Católica del Perú.

8 GARNICA C, RINCÓN J. 2004. Contribución al estudio fitoquímico y de la actividad inmunomoduladora del extracto etanólico de las hojas de Myrcianthes leucoxila. [Tesis]. Universidad nacional de Colombia.

9 OMS. 1958.Métodos de ensayo toxicológico de los aditivos alimentarios. Segundo informe del comité mixto FAO/ OMS de expertos. Ginebra.

10 Silveira A, PAZ L. 2008. New species of baccharis (asteraceae, astereae) from Brazil. Bonplandia; 17(1): 13-24.

11 Salcedo Ortiz L, Sterner O, Almanza V, Giovanna R. 2001. Phytochemical study of Baccharis latifolia L.T., Rev. Boliv. Quim. 18(1):43-46.

12 ABAD M, BERMEJO P. 2007. Baccharis compositae a review update. Arkivoc (7): 79-96.

13 Rojas J, Velazco J, Rojas L, Díaz T, Carmona J, Morales A. 2010. Chemical composition and antibacterial activity of the essential oil of Baccharis latifolia Pers. And B. prunifolia H.B \& K. (Asteraceae). Natural Product Communications. 12(2): 1245-1248. 
14 Massignani J, Lemos M, Maistro E, Schaphouser H, Fabiane R, Barreto J. 2009. Antiulcerogenic activity of the essential oil of Baccharis dracunculifolia on different experimental models in rats. Phytotherapy research. 4: 123-127.

15 Aziz M, Naoual T, Ahmed K, Hassane M, Bnouham M, Abderrahim Z. 2010. Relaxant effect of aqueous extract of Cistus ladaniferus on rodent intestinal contractions. 77: 425-428.

16 Emendorfer F, Bellato F, Noldin V, Neiro R, CEChinel-Filho V. 2008. Evaluation of the relaxant action of some brazilian medicinal plants in isolated guinea-pig ileum and rat duodenum. J. Pharm Pharmaceut Sci. 8(1):63-68.

17 Yoro G, Sarr A, Moctar A, Faye B. 2011. Myorelaxant and antispasmodic effects of the aqueous extracto of Mitragyna inermes barks on wistar rat ileum. El servier. (75): 447-450.

18 Herrera M, Zarzuelo A, Jumenez J, Marhuenda E, Duarte J. 2006. Effects of flavonoids on rat aortic smooth musclecontractility: structure-activity relationships. Gen. Pharmac. 27(2): 273-277.

19 Astudillo A, Mata R, y NAVARrete A. 2009. El reino vegetal, fuente de efectos antiespasmódicos gastrointestinales y antidiarreicos. Rev. Latinoamer. Quím. 37(1): 7-44.

20 Mattson M. 2008. Hormesis defined. Ageing Res Rev. 7(1): 1-7.

21 CAlabrese E. 2008. Hormesis and medicine. Br J Clin Pharmacol. 66(5): 594-617.

22 ARAGADVAY S. 2009. Elaboración y control de calidad de tintura y gel cicatrizante y antiinflamatorio a base de chilca (Baccharis latifolia) y hierbamora (Solanum nigrum) [Tesis]. Escuela superior politécnica de Chimborazo. Ecuador. 